Pré-Publicações do Departamento de Matemática

Universidade de Coimbra

Preprint Number 10-22

\title{
ON THE LAX REPRESENTATION OF THE 2-COMPONENT KP AND 2D TODA HIERARCHIES
}

\author{
GUIDO CARLET AND MANUEL MAÑAS
}

\begin{abstract}
The Lax formulation of the multicomponent KP and 2D Toda hierarchies involves several implicit constraints. We show that, at least in the 2-component case, it is possible to explicitly solve such constraints and identify a set of free dependent variables for such hierarchies.
\end{abstract}

KeYwords: Multicomponent hierarchy, KP, 2D Toda, Lax representation.

AMS Subject Classification (2000): 37K10.

\section{Introduction}

The Kadomtsev-Petviashvili (KP) and the two-dimensional Toda (2D Toda) hierarchies are the most well-known hierarchies associated to $2+1$ integrable systems. They both admit the so-called multicomponent generalizations where the scalar Lax operators are replaced by matrix-valued ones. The multicomponent KP hierarchy has been originally defined by Date, Jimbo, Kashiwara, and Miwa [6] while the multicomponent 2D Toda hierarchy was introduced in the seminal paper [13] by Ueno and Takasaki. They have been further studied by several authors, see e.g. $[3,9,12]$. These hierarchies have been recently the subject of much interest in relation e.g. with multiple orthogonal polynomials [2], Brownian motion [1] and the Givental group action on the space of Frobenius structures [8].

Differently from the scalar case, in the multicomponent hierarchies the matrix Lax operators have to satisfy certain constraints. Let's look first at the usual KP hierarchy. In this case one has a single scalar pseudo-differential Lax operator

$$
L_{K P}=\partial+u_{1} \partial^{-1}+u_{2} \partial^{-2}+\ldots
$$

The commuting flows of the hierarchy are given by the Lax equations

$$
\frac{\partial L_{K P}}{\partial t_{n}}=\left[\left(L_{K P}^{n}\right)_{+}, L_{K P}\right]
$$

Received June 30, 2010. 
where $(\cdot)_{+}$denotes the projection of a pseudo-differential operator to its differential part. Formally the Lax equations might be considered as an algebraic device to produce sequences of differential polynomials

$$
P_{k, n}:=\left(u_{k}\right)_{t_{n}} \in \mathcal{A}_{K P}=\mathbb{C}\left[\left\{u_{k}, u_{k}^{\prime}, u_{k}^{\prime \prime}, \ldots\right\}_{k \geqslant 1}\right]
$$

in the dependent variables $u_{k}, k \geqslant 1$, such that the corresponding derivations

$$
\partial_{t_{n}}:=\sum_{s \geqslant 0, k \geqslant 1}\left(\partial_{x}^{s} P_{k, n}\right) \frac{\partial}{\partial u_{k}^{(s)}}
$$

on the algebra $\mathcal{A}_{K P}$ commute.

In the simple case of the 2-component KP hierarchy, one has two pseudodifferential Lax operators

$$
L=\partial+L_{1} \partial^{-1}+\ldots, \quad C=E_{11}+C_{1} \partial^{-1}+\ldots
$$

where the coefficients are 2 by 2 matrices. Let $\mathcal{A}^{\prime}$ be the algebra of differential polynomials in the entries $\left(L_{i}\right)_{\alpha, \beta},\left(C_{i}\right)_{\alpha, \beta}, \alpha, \beta=1,2$, of the matrix coefficients of $L$ and $C$. As before the Lax equations (6),(7) define sequences of elements in $\mathcal{A}^{\prime}$. However in this case the corresponding derivations don't commute unless an infinite set of differential identities generated by the constraints

$$
C^{2}=C, \quad[L, C]=0
$$

are taken into account. In principle it is not easy to estabilish if these identities can be used to eliminate some variables or if it is possible to write the flows as derivations on a smaller algebra $\mathcal{A}$ of differential polynomials without extra constraints.

In the present work we will show that, at least in the case of the 2component $\mathrm{KP}$ and 2D Toda hierarchies, it is possible to explicitly solve the constraints above and to identify a set of "free" dependent variables.

In the case of the 2-component KP hierarchy the operator $C$ turns out to be parametrized in terms of its off-diagonal part $C_{A}$, e.g. by the formula

$$
C=\sqrt{1+2 C_{A}} E_{11} \sqrt{1+2 C_{A}}
$$

while the operator $L$ is parametrized by its diagonal part $L_{D}$ and by $C_{A}$ by the formula

$$
L=L_{D}-\frac{1}{2} \mathcal{D}\left(\sqrt{1-4 C_{A}^{2}}\right)
$$

where $\mathcal{D}$ is the derivation on the space of formal power series in $C_{A}$ defined by $\mathcal{D}(1)=0$ and $\mathcal{D}\left(C_{A}\right)=H L_{D}$. 
Substituting these formulas in the Lax equations we obtain evolutionary type equations that only involve the variables in $L_{D}$ and $C_{A}$. The Lax equations hence define sequences elements in the algebra $\mathcal{A}$ of differential polynomials in the variables $\left(L_{i}\right)_{11},\left(L_{i}\right)_{22},\left(C_{i}\right)_{12}$ and $\left(C_{i}\right)_{21}$ such that the corresponding derivations on $\mathcal{A}$ commute, without any constraint.

Similar results are proved for the case of the 2-component 2D Toda hierarchy.

One of our main motivations for trying to construct a constraint-free Lax formulation of these multicomponent hierarchies has been the recent construction [5] of an infinite-dimensional Frobenius manifold associated to the dispersionless limit of the usual 2D Toda hierarchy. To see if such a construction is also applicable to the case of multicomponent hierarchies one would like to understand if the costruction of a bi-Hamiltonian structure (along the lines e.g. of [4]) is possible, and if the dispersionless limit for these hierarchies can be understood in the usual way in terms of the symbol of a convenient Lax operator. We hope that giving a formulation free of constraints at least in the 2-component case could be a first step in this program.

An example of 2-component hierarchy in which such program seems to be possible is the case of the 2-component BKP hierarchy where a bihamiltonian structure [14] has been found which could possibily lead to an infinitedimensional Frobenius manifold. However, in this case, one exploits a reformulation [11] of the Lax representation of the hierarchy in terms of two scalar pseudo-differential operators .

This paper is organized in two main sections. In section 2 we consider the 2-component KP hierarchy. The definition of this hierarchy in terms of pseudo-differential operators is recalled. We show that the constraints on the Lax operators can be solved explicitly and we identify the dependent variables of the hierarchy. In Section 3 an analogous construction is developed in the case of the 2-component 2D Toda hierarchy. In this case the algebra of formal difference operators is considered and a similar solution of the constraints is given. Section 4 is devoted to a summary and a discussion of further developments, in particular to the possibility of generalizing the present approach to the $n$-component case.

Notation. We use the following notations

$$
E_{11}=\left(\begin{array}{ll}
1 & 0 \\
0 & 0
\end{array}\right), \quad E_{22}=\left(\begin{array}{cc}
0 & 0 \\
0 & 1
\end{array}\right), \quad H=\left(\begin{array}{cc}
1 & 0 \\
0 & -1
\end{array}\right)
$$


with the identity matrix denoted by 1 .

Recall that the algebra of (formal) pseudo-differential operators is given by formal series in the symbol $\partial$ like

$$
\sum_{k=-\infty}^{n} a_{k} \partial^{k}
$$

with algebra structure defined by

$$
\partial^{k} f=f \partial^{k}+\left(\begin{array}{c}
k \\
1
\end{array}\right) f^{\prime} \partial^{k-1}+\ldots
$$

We will consider the coefficients $a_{k}$ as elements in the algebra of differential polynomials in the dependent variables of the hierarchy. See e.g. [7] for further details on the formal setting.

Analogously, we define the formal difference operators as formal series in $\Lambda$, either of the form

$$
\sum_{k=-\infty}^{n} a_{k} \Lambda^{k} \quad \text { or } \sum_{k=-m}^{\infty} a_{k} \Lambda^{k}
$$

were the algebra structure is given by $\Lambda^{k}=\left(\Lambda^{k} f\right) \Lambda^{k}$. In this case the coefficients $a_{k}$ will be difference polynomials in the dependent variables of the hierarchy. See e.g. [10] for further details.

\section{The 2-component KP hierarchy}

The usual Lax formulation of the 2-component KP hierarchy $[6,9,3]$ is the following. One considers $2 \times 2$-matrix-valued pseudo-differential operators $L$, $C^{(1)}$ and $C^{(2)}$ of the form

$$
\begin{aligned}
L & =\partial+L_{1} \partial^{-1}+L_{2} \partial^{-2}+\cdots, \\
C^{(1)} & =E_{11}+C_{1}^{(1)} \partial^{-1}+C_{2}^{(1)} \partial^{-2}+\cdots, \\
C^{(2)} & =E_{22}+C_{1}^{(2)} \partial^{-1}+C_{2}^{(2)} \partial^{-2}+\cdots,
\end{aligned}
$$

which satisfy the constraints

$$
\begin{aligned}
& C^{(i)} C^{(j)}=\delta_{i j} C^{(i)} \quad i, j=1,2, \\
& C^{(1)}+C^{(2)}=1, \\
& {\left[L, C^{(i)}\right]=0 \quad i=1,2 .}
\end{aligned}
$$


Clearly in this case $C^{(2)}$ can be expressed in terms of $C:=C^{(1)}$ and it is sufficient to consider the constraints

$$
\begin{aligned}
& C^{2}=C, \\
& {[L, C]=0 .}
\end{aligned}
$$

Two sets of flows $t_{i, n}$ for $i=1,2$ and $n \geqslant 0$ are defined by the Lax equations

$$
\begin{aligned}
& \frac{\partial L}{\partial t_{i, n}}=\left[\left(C^{(i)} L^{n}\right)_{+}, L\right], \\
& \frac{\partial C^{(j)}}{\partial t_{i, n}}=\left[\left(C^{(i)} L^{n}\right)_{+}, C^{(j)}\right] .
\end{aligned}
$$

By standard arguments one proves that these flows commute and that they preserve the constraints above.

The Lax equations (6)-(7), however, define commutative flows only if the constraints (4)-(5) are satisfied. We now show that it is possible to explicitly solve these constraints.

We start our analysis from the first constraint (4).

Proposition 1. A $2 \times 2$-matrix-valued pseudo-differential operator $C$ of the form

$$
C=E_{11}+C_{1} \partial^{-1}+\cdots
$$

satisfies the constraint $C^{2}=C$ if and only if it can be written

$$
C=C_{A}+C_{D} \quad \text { with } \quad C_{D}=\frac{1}{2}+\frac{H}{2} \sqrt{1-4 C_{A}^{2}}
$$

where $C_{D}$ and $C_{A}$ are the diagonal and off-diagonal parts of $C$, respectively. Proof. If we split the contraint $C^{2}=C$ in its diagonal and off-diagonal parts we see that it is equivalent to the system

$$
\begin{aligned}
& C_{D}^{2}+C_{A}^{2}=C_{D}, \\
& C_{A} C_{D}+C_{D} C_{A}=C_{A} .
\end{aligned}
$$

Equation (10) is written

$$
\left(C_{D}-\frac{1}{2}\right)^{2}=\frac{1}{4}\left(1-4 C_{A}^{2}\right)
$$

which is solved, taking care of the correct leading term of $C_{D}-\frac{1}{2}=\frac{H}{2}+\cdots$, by the formula (9). Using the fact that $H$ commutes with diagonal matrices and 
anti-commutes with off-diagonal matrices, it is easy to check that formula (9) satisfies (11), too.

The square root appearing in (9) is defined by the usual power-series expansion of $\sqrt{1-x}=1-\frac{x}{2}+\cdots$. This power series gives a well-defined pseudo-differential operator since $C_{A}$ is of order 1 in $\partial^{-1}$, hence only a finite number of terms in the power series contribute to each given order in $\partial^{-1}$.

Corollary 2. The parametrization (9) of $C$ can be written explicitly as

$$
C=\left(\begin{array}{cc}
\frac{1}{2}+\frac{1}{2} \sqrt{1-4 a \tilde{a}} & a \\
\tilde{a} & \frac{1}{2}-\frac{1}{2} \sqrt{1-4 \tilde{a} a}
\end{array}\right)
$$

where $a, \tilde{a}$ are scalar pseudo-differential operators of order $O\left(\partial^{-1}\right)$ :

$$
\begin{aligned}
& a=a_{1} \partial^{-1}+a_{2} \partial^{-2}+a_{3} \partial^{-3}+\cdots, \\
& \tilde{a}=\tilde{a}_{1} \partial^{-1}+\tilde{a}_{2} \partial^{-2}+\tilde{a}_{3} \partial^{-3}+\cdots .
\end{aligned}
$$

We see at once that the entries of the matrix pseudo-differential operator $C$ are expressed as differential polynomials in the coefficients of $a, \tilde{a}$. Recall that the square root of a scalar pseudo-differential operator $b=1+b_{1} \partial^{-1}+\cdots$ can be defined by the power series above or equivalently as the unique operator $\sqrt{b}=1+\tilde{b}_{1} \partial^{-1}+\cdots$ such that $(\sqrt{b})^{2}=b$, where $\tilde{b}_{k}$ are differential polynomials in the $b_{l}$ 's, i.e. $\tilde{b}_{k} \in \mathbb{C}\left[\left\{b_{l}^{(n)}\right\}\right]$.

Example 3. Expanding up to the third order in $\partial^{-1}$ we obtain $C=\left(\begin{array}{cc}1-a_{1} \tilde{a}_{1} \partial^{-2}+\left(a_{1} \tilde{a}_{1}^{\prime}-a_{2} \tilde{a}_{1}-a_{1} \tilde{a}_{2}\right) \partial^{-3} & a_{1} \partial^{-1}+a_{2} \partial^{-2}+a_{3} \partial^{-3} \\ \tilde{a}_{1} \partial^{-1}+\tilde{a}_{2} \partial^{-2}+\tilde{a}_{3} \partial^{-3} & a_{1} \tilde{a}_{1} \partial^{-2}-\left(\tilde{a}_{1} a_{1}^{\prime}-\tilde{a}_{2} a_{1}-\tilde{a}_{1} a_{2}\right) \partial^{-3}\end{array}\right)+O\left(\partial^{-4}\right)$

Remark 4. Using the fact that $E_{11}=\frac{1+H}{2}$ and $H$ anti-commutes with $C_{A}$ it is possible to rewrite the formula (9) in the interesting form

$$
C=\sqrt{1+2 C_{A}} E_{11} \sqrt{1+2 C_{A}} .
$$

From this expression it is easy to check that the constraint $C^{2}=C$ is satisfied and that $C$ has the correct leading term as in (8).

We now consider the second constraint (5). We first introduce

Definition 5. The operator $\mathcal{D}$ denotes the derivation on the space of formal power series in the variable $C_{A}$ defined by $\mathcal{D}(1)=0$ and $\mathcal{D}\left(C_{A}\right)=H L_{D}$, and $L_{D}$ and $L_{A}$ denote the diagonal and off-diagonal parts of $L$, respectively, 
and consider the following lemmas:

Lemma 6. The constraint (5) is equivalent to

$$
\begin{aligned}
& {\left[L_{A}, C_{A}\right]=-\left[L_{D}, C_{D}\right]} \\
& {\left[L_{A}, C_{D}\right]=-\left[L_{D}, C_{A}\right]}
\end{aligned}
$$

holds.

Proof. Consider the diagonal and off-diagonal parts of $[L, C]=0$.

Lemma 7. The following identity holds

$$
\operatorname{ad}_{H L_{D}}=-\operatorname{ad}_{C_{A}} \circ \mathcal{D}
$$

when acting on power series in the variable $C_{A}$.

Proof. It follows from the definition of the derivation $\mathcal{D}$ and the the fact that $\operatorname{ad}_{C_{A}}$ is identically zero when acting on such series, hence $0=\mathcal{D} \circ \operatorname{ad}_{C_{A}}=$ $\operatorname{ad}_{\mathcal{D}\left(C_{A}\right)}+\operatorname{ad}_{C_{A}} \circ \mathcal{D}$.

Lemma 8. Let $C=C^{2}$ as in Proposition 1. Then the constraint (14) is equivalent to the identity

$$
\operatorname{ad}_{L_{A}}=-\frac{1}{2} \operatorname{ad}_{\mathcal{D}\left(\sqrt{1-4 C_{A}^{2}}\right)}
$$

on power series in $C_{A}$.

Proof. Indeed from (14) and (9) we have

$$
\operatorname{ad}_{L_{A}} C_{A}=-\frac{1}{2} \operatorname{ad}_{H L_{D}}\left(\sqrt{1-4 C_{A}^{2}}\right)
$$

and, using (16)

$$
\begin{aligned}
\operatorname{ad}_{L_{A}} C_{A} & =\frac{1}{2} \operatorname{ad}_{C_{A}} \circ \mathcal{D}\left(\sqrt{1-4 C_{A}^{2}}\right) \\
& =-\frac{1}{2} \operatorname{ad}_{\mathcal{D}\left(\sqrt{1-4 C_{A}^{2}}\right)} C_{A} .
\end{aligned}
$$


Proposition 9. Let $C=C^{2}$ be as in the Proposition 1. A $2 \times 2$-matrixvalued pseudo-differential operator $L$ of the form (1) commutes with $C$ if and only if it has the form

$$
L=L_{A}+L_{D} \quad \text { with } \quad L_{A}=-\frac{1}{2} \mathcal{D}\left(\sqrt{1-4 C_{A}^{2}}\right) .
$$

Proof. Consider (15), which can be easily rewritten as

$$
L_{A} H \sqrt{1-4 C_{A}^{2}}+\frac{H}{2} \operatorname{ad}_{L_{A}}\left(\sqrt{1-4 C_{A}^{2}}\right)=-\left[L_{D}, C_{A}\right] .
$$

Using formula (17) we compute

$$
\begin{aligned}
\operatorname{ad}_{L_{A}}\left(\sqrt{1-4 C_{A}^{2}}\right) & =-\frac{1}{2} \operatorname{ad}_{\mathcal{D}\left(\sqrt{1-4 C_{A}^{2}}\right)}\left(\sqrt{1-4 C_{A}^{2}}\right) \\
& =-\mathcal{D}\left(\sqrt{1-4 C_{A}^{2}}\right) \sqrt{1-4 C_{A}^{2}}+\frac{1}{2} \mathcal{D}\left(1-4 C_{A}^{2}\right) .
\end{aligned}
$$

Inserting this formula in (19) we obtain

$$
L_{A} H \sqrt{1-4 C_{A}^{2}}-\frac{H}{2} \mathcal{D}\left(\sqrt{1-4 C_{A}^{2}}\right) \sqrt{1-4 C_{A}^{2}}-H \mathcal{D}\left(C_{A}^{2}\right)=-\left[L_{D}, C_{A}\right] .
$$

The last two terms in the last expression cancel and, since the square root is an invertible power series, we obtain (18).

On the other hand inserting (18) and (9) in (14) we see that this equation is satisfied if and only if

$$
\operatorname{ad}_{C_{A}} \circ \mathcal{D}\left(\sqrt{1-4 C_{A}^{2}}\right)=-\operatorname{ad}_{H L_{D}}\left(\sqrt{1-4 C_{A}^{2}}\right)
$$

which clearly follows from (16). Equations (18) and (9) solve also (15); indeed,

$$
\begin{aligned}
{\left[L_{A}, C_{D}\right] } & =-\frac{1}{4}\left[\mathcal{D}\left(\sqrt{1-4 C_{A}^{2}}\right), H \sqrt{1-4 C_{A}^{2}}\right] \\
& =\mathcal{D}\left(C_{A}^{2}\right) H \\
& =-\left[L_{D}, C_{A}\right] .
\end{aligned}
$$

The Proposition is proved.

Corollary 10. The matrix L has the following explicit parametrization $L=\left(\begin{array}{cc}l & \frac{1}{2} \sum_{n=0}^{\infty} c_{n} \sum_{s=1}^{n}(a \tilde{a})^{s-1}(a \tilde{l}-l a)(\tilde{a} a)^{n-s} \\ -\frac{1}{2} \sum_{n=0}^{\infty} c_{n} \sum_{s=1}^{n}(\tilde{a} a)^{s-1}(\tilde{a} l-\tilde{l} \tilde{a})(a \tilde{a})^{n-s}\end{array}\right)$ 
where $l, \tilde{l}$ are pseudo-differential operators of the form $\partial+O\left(\partial^{-1}\right)$

$$
\begin{aligned}
& l=\partial+l_{1} \partial^{-1}+l_{2} \partial^{-2}+\cdots, \\
& \tilde{l}=\partial+\tilde{l}_{1} \partial^{-1}+\tilde{l}_{2} \partial^{-2}+\cdots,
\end{aligned}
$$

while $a, \tilde{a}$ are the off-diagonal entries of $C_{A}$ defined in (12)-(13) and the coefficients $c_{n}$ are defined by the expansion $\sqrt{1-4 x^{2}}=\sum_{n=0}^{\infty} c_{n} x^{2 n}$.

Example 11. Expanding we obtain

$L=\left(\begin{array}{cc}\partial+l_{1} \partial^{-1}+l_{2} \partial^{-2} & a_{1}^{\prime} \partial^{-1}+\left(a_{2}^{\prime}+a_{1}\left(l_{1}-\tilde{l}_{1}\right)\right) \partial^{-2} \\ -\tilde{a}_{1}^{\prime} \partial^{-1}-\left(\tilde{a}_{2}^{\prime}+\tilde{a}_{1}\left(\tilde{l}_{1}-l_{1}\right)\right) \partial^{-2} & \partial+\tilde{l}_{1} \partial^{-1}+\tilde{l}_{2} \partial^{-2}\end{array}\right)+O\left(\partial^{-3}\right)$.

Indeed $L$ can be expressed only in terms of its diagonal coefficients, i.e. $l_{i}$, $\tilde{l}_{i}$, and the off-diagonal coefficients of $C$, i.e. $a_{i}, \tilde{a}_{i}$.

Remark 12. The parametrization (18) can also be written in the form

$$
L=-\sqrt{1+2 C_{A}} E_{11} \mathcal{D}\left(\sqrt{1-2 C_{A}}\right)-\mathcal{D}\left(\sqrt{1+2 C_{A}}\right) E_{22} \sqrt{1-2 C_{A}}
$$

as one can easily check.

Note that the dependence of $L$ on $L_{D}$ is linear, while $L$ and $C$ depend on $C_{A}$ non-linearly.

Using the results obtained above with find the following formulation of the 2 -component KP hierarchy, which is free of constraints. The dependent variables are organized in two 2 by 2 matrix valued pseudo-differential operators of the form

$$
\begin{aligned}
& L_{D}=\partial+L_{D, 1} \partial^{-1}+L_{D, 2} \partial^{-2}+\cdots, \\
& C_{A}=C_{A, 1} \partial^{-1}+C_{A, 2} \partial^{-2}+\cdots,
\end{aligned}
$$

where $L_{D}$ is diagonal and $C_{A}$ off-diagonal. We now introduce

Definition 13. The differential operators $B_{1, n}$ and $B_{2, n}$ are given by

$$
\begin{aligned}
& B_{1, n}=\left[\left(\frac{1}{2}+C_{A}+\frac{H}{2} \sqrt{1-4 C_{A}^{2}}\right)\left(L_{D}-\frac{1}{2} \mathcal{D}\left(\sqrt{1-4 C_{A}^{2}}\right)\right)^{n}\right]_{+} \\
& B_{2, n}=\left[\left(\frac{1}{2}-C_{A}-\frac{H}{2} \sqrt{1-4 C_{A}^{2}}\right)\left(L_{D}-\frac{1}{2} \mathcal{D}\left(\sqrt{1-4 C_{A}^{2}}\right)\right)^{n}\right]_{+}
\end{aligned}
$$

and $(\cdot)_{D},(\cdot)_{A}$ denote the projections on the diagonal and off the diagonal respectively. 
Proposition 14. The equations of the hierarchy are

$$
\begin{aligned}
& \frac{\partial L_{D}}{\partial t_{i, n}}=\left[\left(B_{i, n}\right)_{D}, L_{D}\right]-\frac{1}{2}\left[\left(B_{i, n}\right)_{A}, \mathcal{D}\left(\sqrt{1-4 C_{A}^{2}}\right)\right], \\
& \frac{\partial C_{A}}{\partial t_{i, n}}=\left[\left(B_{i, n}\right)_{D}, C_{A}\right]+\frac{1}{2}\left[\left(B_{i, n}\right)_{A}, H \sqrt{1-4 C_{A}^{2}}\right] .
\end{aligned}
$$

Proof. This result is obtained by projection of the equations (6) and (7) on the diagonal and off the diagonal respectively.

2.1. Examples and the Davey-Stewartson system. Recalling the formulae for

$$
L_{A}=-\frac{1}{2} \mathcal{D}\left(\sqrt{1-4 C_{A}^{2}}\right) \quad \text { and } \quad C_{D}=\frac{1}{2}+\frac{H}{2} \sqrt{1-4 C_{A}^{2}}
$$

we get

$$
L_{A}=L_{A, 1} \partial^{-1}+L_{A, 2} \partial^{-2}+L_{A, 3} \partial^{-3}+\cdots
$$

with the first three coefficients given by

$$
\begin{aligned}
L_{A, 1} & =H\left(C_{A, 1}\right)_{x} \\
L_{A, 2}=H\left(\left(C_{A, 2}\right)_{x}+\left[L_{D, 1}, C_{A, 1}\right]\right) & \\
L_{A, 3}=H\left(\left(C_{A, 3}\right)_{x}+\left[L_{D, 1}, C_{A, 2}\right]\right. & +\left[L_{D, 2}, C_{A, 1}\right]-L_{D, 1}\left(C_{A, 1}\right)_{x}+C_{A, 1}\left(L_{D, 1}\right)_{x} \\
& \left.+\left(C_{A, 1}\right)_{x} C_{A, 1}^{2}+C_{A, 1}^{2}\left(C_{A, 1}\right)_{x}-C_{A, 1}\left(C_{A, 1}\right)_{x} C_{A, 1}\right) .
\end{aligned}
$$

We also have

$$
C_{D}=E_{11}+C_{D, 2} \partial^{-2}+C_{D, 3} \partial^{-3}+\cdots
$$

with

$$
\begin{aligned}
& C_{D, 2}=-H C_{A, 1}^{2}, \\
& C_{D, 3}=-H\left(C_{A, 1} C_{A, 2}+C_{A, 2} C_{A, 1}-C_{A, 1}\left(C_{A, 1}\right)_{x}\right) .
\end{aligned}
$$


To find the explicit form of the flows we proceed with the computation of the expressions

$$
\begin{array}{ll}
\left(B_{1,1}\right)_{D}=E_{11} \partial, & \left(B_{1,1}\right)_{A}=C_{A, 1} \\
\left(B_{1,2}\right)_{D}=E_{22} \partial, & \left(B_{1,2}\right)_{A}=-C_{A, 1} \\
\left(B_{2,1}\right)_{D}=E_{11} \partial^{2}+2 L_{D, 1} E_{11}-H C_{A, 1}^{2}, & \left(B_{2,1}\right)_{A}=C_{A, 1} \partial+2 E_{11}\left(C_{A, 1}\right)_{x}+C_{A, 2} \\
\left(B_{2,2}\right)_{D}=E_{22} \partial^{2}+2 L_{D, 1} E_{22}+H C_{A, 1}^{2}, & \left(B_{2,2}\right)_{A}=-C_{A, 1} \partial-2 E_{22}\left(C_{A, 1}\right)_{x}-C_{A, 2} .
\end{array}
$$

Inserting in the Lax equations (20)-(21) we obtain, for the first couple of times $t_{1,1}, t_{1,2}$, the following equations for $L_{D, 1}$ and $L_{D, 2}$

$$
\begin{aligned}
& \partial_{1,1} L_{D, 1}=\left(E_{11} L_{D, 1}-H\left(C_{A, 1}\right)^{2}\right)_{x} \\
& \partial_{1,2} L_{D, 1}=\left(E_{22} L_{D, 1}+H\left(C_{A, 1}\right)^{2}\right)_{x} \\
& \partial_{1,1} L_{D, 2}=E_{11}\left(L_{D, 2}\right)_{x}+H\left(\left(\left(C_{A, 1}\right)_{x}\right)^{2}-C_{A, 1}\left(C_{A, 2}\right)_{x}-\left(C_{A, 2}\right)_{x} C_{A, 1}\right), \\
& \partial_{1,2} L_{D, 2}=E_{22} L_{D, 2, x}-H\left(\left(\left(C_{A, 1}\right)_{x}\right)^{2}-C_{A, 1}\left(C_{A, 2}\right)_{x}-\left(C_{A, 2}\right)_{x} C_{A, 1}\right),
\end{aligned}
$$

while for $C_{A, 1}$ and $C_{A, 2}$ we have

$$
\begin{aligned}
& \partial_{1,1} C_{A, 1}=E_{11}\left(C_{A, 1}\right)_{x}+\left[E_{11}, C_{A, 2}\right], \\
& \partial_{1,2} C_{A, 1}=E_{22}\left(C_{A, 1}\right)_{x}+\left[E_{22}, C_{A, 2}\right], \\
& \partial_{1,1} C_{A, 2}=E_{11}\left(C_{A, 2}\right)_{x}+\left[E_{11}, C_{A, 3}\right]+2 H\left(C_{A, 1}\right)^{3}, \\
& \partial_{1,2} C_{A, 2}=E_{22}\left(C_{A, 2}\right)_{x}+\left[E_{11}, C_{A, 3}\right]-2 H\left(C_{A, 1}\right)^{3} .
\end{aligned}
$$

Combining some equations of the hierarchy we can obtain an example of $2+1$ equation related to this hierarchy. Indeed, from the equations for $C_{A, 1}$ and $C_{A, 2}$ we can derive $C_{A, 2}$ and $C_{A, 3}$ in terms of $C_{A, 1}$ and its $x, t_{1, i}$ derivatives, $i=1,2$. For that aim is enough to observe that $\operatorname{ad}_{E_{i i}}^{2}=\mathrm{id}, i=1,2$, over the off-diagonal matrices. Hence,

$$
\begin{aligned}
C_{A, 2} & =\left[E_{11},\left(\partial_{1,1}-E_{11} \partial\right) C_{A, 1}\right]=\left[E_{22},\left(\partial_{1,2}-E_{22} \partial\right) C_{A, 1}\right] \\
C_{A, 3} & =\left[E_{11},\left(\partial_{1,1}-E_{11} \partial\right)^{2} C_{A, 1}\right]-\left(C_{A, 1}\right)^{3}=\left[E_{22},\left(\partial_{1,2}-E_{22} \partial\right)^{2} C_{A, 1}\right]+\left(C_{A, 1}\right)^{3} .
\end{aligned}
$$


We now write the $t_{2, i}$-flows, $i=1,2$, but only for $L_{D, 1}$

$$
\begin{aligned}
\partial_{2,1} L_{D, 1} & =E_{11}\left(L_{D, 1, x x}+2 L_{D, 2, x}\right)-H\left(C_{A, 1}\left(C_{A, 1}\right)_{x x}-\left(\left(C_{A, 1}\right)_{x}\right)^{2}+\left(C_{A, 1} C_{A, 2}\right)_{x}\right. \\
& \left.-2\left[E_{11}\left(C_{A, 1}\right)_{x}, E_{22}\left(C_{A, 1}\right)_{x}\right]+C_{A, 1}\left[L_{D, 1},\left(C_{A, 1}\right)_{x}\right]+\left[L_{D, 1}, A_{1, x}\right] C_{A, 1}\right), \\
\partial_{2,2} L_{D, 1} & =E_{22}\left(L_{D, 1, x x}+2 L_{D, 2, x}\right)+H\left(C_{A, 1}\left(C_{A, 1}\right)_{x x}-\left(\left(C_{A, 1}\right)_{x}\right)^{2}+\left(C_{A, 1} C_{A, 2}\right)_{x}\right. \\
& \left.-2\left[E_{22}\left(C_{A, 1}\right)_{x}, E_{11}\left(C_{A, 1}\right)_{x}\right]+C_{A, 1}\left[L_{D, 1},\left(C_{A, 1}\right)_{x}\right]+\left[L_{D, 1}, A_{1, x}\right] C_{A, 1}\right)
\end{aligned}
$$

and $C_{A, 1}$

$$
\begin{aligned}
& \partial_{2,1} C_{A, 1}=E_{11}\left(A_{1, x x}+2\left(C_{A, 2}\right)_{x}\right)+\left[E_{11}, C_{A, 3}\right]+2\left[L_{D, 1} E_{11}, C_{A, 1}\right], \\
& \partial_{2,2} C_{A, 1}=E_{22}\left(A_{1, x x}+2\left(C_{A, 1}\right)_{x}\right)+\left[E_{22}, C_{A, 3}\right]+2\left[L_{D, 1} E_{22}, C_{A, 1}\right] .
\end{aligned}
$$

Substituting (26)-(29) and (22)-(23) in the above equations results into

$$
\begin{aligned}
& \partial_{2,1} C_{A, 1}=H\left(\partial_{1,1}^{2} C_{A, 1}-2\left(C_{A, 1}\right)^{3}\right)+2\left[L_{D, 1} E_{11}, C_{A, 1}\right], \\
& \partial_{2,2} C_{A, 1}=-H\left(\partial_{1,2}^{2} C_{A, 1}-2\left(C_{A, 1}\right)^{3}\right)+2\left[L_{D, 1} E_{22}, C_{A, 1}\right], \\
& \left(E_{11} \partial_{1,1}+E_{22} \partial_{1,2}\right)\left(L_{D, 1}\right)=\left(\left(C_{A, 1}\right)^{2}\right)_{x} .
\end{aligned}
$$

Now, if we write

$$
L_{D, 1}=\left(\begin{array}{cc}
U & 0 \\
0 & V
\end{array}\right), \quad C_{A, 1}=\left(\begin{array}{cc}
0 & p \\
q & 0
\end{array}\right)
$$

we get the Davey-Stewartson system

$$
\begin{aligned}
\partial_{2,1} p & =\partial_{1,1}^{2} p-2 p^{2} q+2 U p, \\
\partial_{2,1} q & =-\partial_{1,1}^{2} q-2 p q^{2}-2 U q, \\
\partial_{2,2} p & =-\partial_{1,1}^{2} p+2 p^{2} q-2 V p, \\
\partial_{2,2} q & =\partial_{1,1}^{2} q+2 p q^{2}+2 V q, \\
\partial_{1,1} U & =\partial_{1,2} V=(p q)_{x} .
\end{aligned}
$$

\section{The 2-component $2 \mathrm{D}$ Toda hierarchy}

In this section we show that a similar analysis can be performed for the 2-component 2D Toda hierarchy. In this case one has to deal with matrixvalued formal difference operators. 
The 2-component 2D Toda hierarchy $[13,12]$ is defined in terms of formal Lax difference operators $L, \bar{L}, C^{(i)}, \bar{C}^{(i)}$ for $i=1,2$ of the form

$$
\begin{aligned}
L & =\Lambda+L_{0}+L_{-1} \Lambda^{-1}+\cdots, \\
\bar{L} & =\bar{L}_{-1} \Lambda^{-1}+\bar{L}_{0}+\bar{L}_{1} \Lambda+\cdots, \\
C^{(i)} & =E_{i i}+C_{1}^{(i)} \Lambda^{-1}+\cdots, \\
\bar{C}^{(i)} & =\bar{C}_{0}^{(i)}+\bar{C}_{1}^{(i)} \Lambda+\cdots,
\end{aligned}
$$

where $\bar{L}_{-1}$ is invertible and $\bar{C}_{0}^{(i)}$ is similar to $E_{i i}$. These operators are required to satisfy the following constraints

$$
\begin{aligned}
& C^{(i)} C^{(j)}=\delta_{i j} C^{(i)}, \quad i, j=1,2, \\
& \bar{C}^{(i)} \bar{C}^{(j)}=\delta_{i j} \bar{C}^{(i)}, \quad i, j=1,2, \\
& C^{(1)}+C^{(2)}=1=\bar{C}^{(1)}+\bar{C}^{(2)}, \\
& {\left[L, C^{(i)}\right]=0=\left[\bar{L}, \bar{C}^{(i)}\right], \quad i=1,2,}
\end{aligned}
$$

which in this case reduce to

$$
\begin{array}{lll}
C^{2}=C, & {[L, C]=0,} \\
\bar{C}^{2}=\bar{C}, & {[\bar{L}, \bar{C}]=0,}
\end{array}
$$

where we have denoted $C:=C^{(1)}$ and $\bar{C}:=\bar{C}^{(1)}$.

Four sequences of flows $t_{i, n}, \bar{t}_{i, n}$ for $i=1,2$ and $n \geqslant 0$ are defined by

$$
\begin{gathered}
\frac{\partial}{\partial t_{i, n}} \cdot=\left[\left(C^{(i)} L^{n}\right)_{+}, \cdot\right], \\
\frac{\partial}{\partial \bar{t}_{i, n}} \cdot=\left[\left(\bar{C}^{(i)} \bar{L}^{n}\right)_{-}, \cdot\right],
\end{gathered}
$$

where $\cdot$ can be any of the Lax operators $L, \bar{L}, C^{(i)}, \bar{C}^{(i)}$ for $i=1,2$. Again by standard arguments these flows are seen to commute and preserve the constraints above. In particular it is easy to see that the matrices $\bar{C}_{0}^{(j)}$ evolve like

$$
\frac{\partial \bar{C}_{0}^{(j)}}{\partial t_{i, n}}=\left[\operatorname{Res}\left(C^{(i)} L^{n}\right), \bar{C}_{0}^{(j)}\right], \quad \frac{\partial \bar{C}_{0}^{(j)}}{\partial \bar{t}_{i, n}}=\left[-\operatorname{Res}\left(\bar{C}^{(i)} \bar{L}^{n}\right), \bar{C}_{0}^{(j)}\right]
$$

hence the condition of similarity of $\bar{C}_{0}^{(i)}$ and $E_{i i}$ is preserved. 
The solution to the constraints (32) for the operators $L$ and $C$ is completely analogous to that for the 2-component KP hierarchy. We summarize the statement here:

Proposition 15. (a) A $2 \times 2$-matrix-valued formal difference operator $C$ of the form

$$
C=E_{11}+C_{1} \Lambda^{-1}+\ldots
$$

satisfies the constraint $C^{2}=C$ if and only if it can be written

$$
C=C_{A}+C_{D} \quad \text { with } \quad C_{D}=\frac{1}{2}+\frac{H}{2} \sqrt{1-4 C_{A}^{2}}
$$

where $C_{D}$ and $C_{A}$ are the diagonal and off-diagonal parts of $C$, respectively.

(b) Let $C=C^{2}$ be as above. A $2 \times 2$-matrix-valued formal difference operator $L$ of the form (30) commutes with $C$ if and only if it has the form

$$
L=L_{A}+L_{D} \quad \text { with } \quad L_{A}=-\frac{1}{2} \mathcal{D}\left(\sqrt{1-4 C_{A}^{2}}\right),
$$

where $\mathcal{D}$ is the derivation on the space of formal power series in the variable $C_{A}$ defined by $\mathcal{D}(1)=0$ and $\mathcal{D}\left(C_{A}\right)=H L_{D}$, and $L_{D}$ and $L_{A}$ denote the diagonal and off-diagonal parts of $L$, respectively.

To solve the constraints (33) for the operators $\bar{L}$ and $\bar{C}$ we need to take care of the fact that their leading orders are not constant matrices.

The leading order $\bar{C}_{0}$ of the operator $\bar{C}$ is required to be similar to $E_{11}$ and to satisfy $\bar{C}_{0}^{2}=\bar{C}_{0}$; such requirements are equivalent to the following constraints on entries of $\bar{C}_{0}$ :

$$
\bar{C}_{0}=\left(\begin{array}{ll}
a & b \\
c & d
\end{array}\right), \quad\left\{\begin{array}{l}
a+d=1, \\
a d=b c
\end{array}\right.
$$

For simplicity in the following we consider the generic case where $a \neq 0,1$ and we parametrize $\bar{C}_{0}$ as a function of $b=e^{u}, c=e^{v}$ as follows

$$
\bar{C}_{0}=\left(\begin{array}{cc}
w & e^{u} \\
e^{v} & 1-w
\end{array}\right)
$$

where $w=a$ is a fixed choice of a root the quadratic equation above, i.e.

$$
w=\frac{1}{2}\left(1 \pm \sqrt{1-4 e^{u+v}}\right) .
$$


Denote

$$
\phi:=\bar{C}_{0}-E_{22}=\left(\begin{array}{cc}
w & e^{u} \\
e^{v} & -w
\end{array}\right) .
$$

Lemma 16. The matrix $\phi$ is invertible, with $\phi^{-1}=\frac{1}{w} \phi$ and

$$
\bar{C}_{0}=\phi E_{11} \phi^{-1} \text {. }
$$

Proof. Clearly

$$
\operatorname{det} \phi=-w \neq 0
$$

and

$$
\bar{C}_{0} \phi=\bar{C}_{0}\left(\bar{C}_{0}-E_{22}\right)=\bar{C}_{0} E_{11}=\left(\bar{C}_{0}-E_{22}\right) E_{11}=\phi E_{11}
$$

Using the matrix $\phi$ to dress $\bar{C}$ we obtain the parametrization of the first constraint in (33).

Proposition 17. A $2 \times 2$-matrix-valued formal difference operator $\bar{C}$ of the form

$$
\bar{C}=\bar{C}_{0}+\bar{C}_{1} \Lambda+\ldots
$$

where $\bar{C}_{0}$ is of the form (36) and such that it satisfies the constraint $\bar{C}^{2}=\bar{C}$ can be parametrized in terms of $e^{u}, e^{v}$ and of an off-diagonal operator $\hat{C}_{A}$ of the form

$$
\hat{C}_{A}=\hat{C}_{A, 1} \Lambda+\hat{C}_{A, 2} \Lambda^{2}+\ldots
$$

by the formula

$$
\bar{C}=\sqrt{1+2 \tilde{C}} \bar{C}_{0} \sqrt{1+2 \tilde{C}}
$$

or equivalently as

$$
\bar{C}=\tilde{C}+\frac{1}{2}+\left(\bar{C}_{0}-\frac{1}{2}\right) \sqrt{1-4 \tilde{C}^{2}},
$$

where $\tilde{C}:=\left(\bar{C}_{0}-E_{22}\right) \hat{C}_{A} \frac{1}{w}\left(\bar{C}_{0}-E_{22}\right)$.

Proof. The operator $\hat{C}:=\phi^{-1} \bar{C} \phi$ clearly satisfies $\hat{C}^{2}=\hat{C}$ and has the leading term $E_{11}$. Hence one proves as before that

$$
\hat{C}=\hat{C}_{A}+\frac{1}{2}+\frac{H}{2} \sqrt{1-4 \hat{C}_{A}^{2}}
$$

or, as in Remark 4

$$
\hat{C}=\sqrt{1+2 \hat{C}_{A}} E_{11} \sqrt{1+2 \hat{C}_{A}} .
$$


Dressing with $\phi$ one obtains the desired result.

Now consider the leading term of $\bar{L}$ which must satisfy

$$
\bar{L}_{-1} \Lambda^{-1} \bar{C}_{0}=\bar{C}_{0} \bar{L}_{-1}
$$

equivalenty

$$
\left[\phi^{-1} \bar{L}_{-1} \Lambda^{-1} \phi, E_{11}\right]=0 .
$$

Hence $\bar{L}_{-1}$ must be of the form

$$
\bar{L}_{-1}=\phi D \Lambda^{-1} \phi^{-1}
$$

where $D$ is a diagonal matrix.

Clearly the operator $\hat{L}:=\phi^{-1} \bar{L} \phi$ commutes with $\hat{C}$ and has diagonal leading term $D \Lambda^{-1}$. One can easily show, as in the previous cases, that $\hat{L}$ has to be of the form

$$
\hat{L}=\hat{L}_{D}-\frac{1}{2} \mathcal{D}_{H \hat{L}_{D}}\left(\sqrt{1-4 \hat{C}_{A}^{2}}\right) .
$$

Dressing with $\phi$ one obtains the required result:

Proposition 18. Let $\bar{C}=\bar{C}^{2}$ be as in the previous Proposition. $A 2 \times 2$ matrix-valued formal difference operator $\bar{L}$ of the form (31) that commutes with $\bar{C}$ can be parametrized by $\bar{C}$, i.e. by $e^{u}$, $e^{v}$ and $\hat{C}_{A}$, and by a diagonal formal difference operator of the form

$$
\hat{L}_{D}=D \Lambda^{-1}+\hat{L}_{D, 0}+\ldots
$$

by the formula

$$
\bar{L}=\tilde{L}-\frac{1}{2} \hat{\mathcal{D}}\left(\sqrt{1-4 \tilde{C}^{2}}\right)
$$

where $\tilde{L}:=\left(\bar{C}_{0}-E_{22}\right) \hat{L}_{D} \frac{1}{w}\left(\bar{C}_{0}-E_{22}\right)$ and $\hat{\mathcal{D}}$ is the derivation on the space of formal power series in $\tilde{C}$ defined by $\hat{\mathcal{D}}(1)=0$ and

$$
\hat{\mathcal{D}}(\tilde{C})=\left(\bar{C}_{0}-E_{22}\right) H \hat{L}_{D} \frac{1}{w}\left(\bar{C}_{0}-E_{22}\right) .
$$

To conclude, note that the dependent variables of the 2-component $2 \mathrm{D}$ Toda hierarchy are the entries of $C_{A}, L_{D}, \hat{C}_{A}, \hat{L}_{D}$ and the variables $e^{u}, e^{v}$ that parametrize the leading term $\bar{C}_{0}$. 


\section{Concluding remarks}

In this paper we have shown that it is possible to explicitly solve the constraints in the Lax definition of the 2-component KP and 2D Toda hierarchies. It turns out that the KP Lax operators are parametrized by simple formulas like (9) and (18), and that analogous formulas hold in the 2D Toda case. This allows us to identify a set of "free" dependent variables for such hierarchies, which are given in the KP case by the off-diagonal part of the operator $C$ and by the diagonal part of the operator $L$.

We hope that this result will help in the study of reductions, in the construction of bihamiltonian structures and will also clarify the problem of the existence of the dispersionless limit, especially at the level of Lax equations.

We plan to consider the $n$-component case in a subsequent publication. In this regard note that, while the present approach seems to be largely dependent on the properties of 2 by 2 matrices, it is indeed possibile to generalize straighforwardly some results, e.g. Proposition 1, to the case of $n$ by $n$ matrices, by splitting the matrices in four blocks and considering the decomposition in diagonal and off-diagonal blocks. We hope that this property could be exploited to solve also the other constraints that are present in the $n$-component theory.

\section{Acknowledgements}

G. Carlet wishes to thank the Department of Theoretical Physics II of the Universidad Complutense de Madrid for its hospitality and acknowledges the support of ESF-MISGAM exchange grant n. 2326. M. Mañas acknowledges the ESF-MISGAM project and the Spanish Ministerio de Ciencia e Innovación, research project FIS2008-00200.

\section{References}

[1] Adler, Mark; van Moerbeke, Pierre; Vanderstichelen, Didier. Non-intersecting Brownian motions leaving from and going to several points. Preprint arXiv:1005.1303

[2] Álvarez-Fernández, Carlos; Fidalgo Prieto, Ulises; Mañas, Manuel. Multiple orthogonal polynomials of mixed type: Gauss-Borel factorization and the multi-component 2D Toda hierarchy. Preprint arxiv:1004.3916

[3] Bergvelt, M. J.; ten Kroode, A. P. E. Partitions, vertex operator constructions and multicomponent KP equations. Pacific J. Math. 171 (1995), no. 1, 23-88.

[4] Carlet, Guido The Hamiltonian structures of the two-dimensional Toda lattice and R-matrices. Lett. Math. Phys. 71 (2005), no. 3, 209-226. 
[5] Carlet, Guido; Dubrovin, Boris; Mertens, Luca Philippe. Infinite-dimensional Frobenius manifolds for $2+1$ integrable systems. Mathematische Annalen (2010). DOI: 10.1007/s00208-0100509-3.

[6] Date, Etsuro; Jimbo, Michio; Kashiwara, Masaki; Miwa, Tetsuji. Transformation groups for soliton equations. III. Operator approach to the Kadomtsev-Petviashvili equation. J. Phys. Soc. Japan 50 (1981), no. 11, 3806-3812.

[7] Dickey, L. A. Soliton equations and Hamiltonian systems. Second edition. Advanced Series in Mathematical Physics, 26. World Scientific Publishing Co., Inc., River Edge, NJ, 2003. xii +408 pp.

[8] Feigin, Evgeny; van de Leur, Johan; Shadrin, Sergey. Givental symmetries of Frobenius manifolds and multi-component KP tau-functions. Preprint arXiv:0905.0795

[9] Kac, V. G.; van de Leur, J. W. The $n$-component KP hierarchy and representation theory. Integrability, topological solitons and beyond. J. Math. Phys. 44 (2003), no. 8, 3245-3293.

[10] Kuperschmidt, B. A. Discrete Lax equations and differential-difference calculus. Astérisque No. 123 (1985), 212 pp.

[11] Liu, Si-Qi; Wu, Chao-Zhong; Zhang, Youjin. On the Drinfeld-Sokolov hierarchies of D type. Preprint arXiv:0912.5273

[12] Mañas, Manuel; Martínez Alonso, Luis; Álvarez-Fernández, Carlos. The multicomponent 2D Toda hierarchy: discrete flows and string equations. Inverse Problems 25 (2009), no. 6, 065007, $31 \mathrm{pp}$.

[13] Ueno, Kimio; Takasaki, Kanehisa. Toda lattice hierarchy. Group representations and systems of differential equations (Tokyo, 1982), 1-95, Adv. Stud. Pure Math., 4, North-Holland, Amsterdam, 1984.

[14] Xu, Dingdian; Wu, Chao-Zhong. Bihamiltonian structure of the two-component KadomtsevPetviashvili hierarchy of type B. Preprint arXiv:1001.412

Guido CARLet

CMUC, Centre for Mathematics, University of Coimbra, Portugal.

E-mail address: gcarlet@mat.uc.pt

ManUel MaÑAS

Departamento de Fisica Teorica II, Universidad Complutense, Spain.

E-mail address: manuel.manas@fis.ucm.es 